\title{
ReStNeuMap: a tool for automatic extraction of resting-state functional MRI networks in neurosurgical practice
}

\author{
Domenico Zacà, PhD, ${ }^{1}$ Jorge Jovicich, PhD, ${ }^{1}$ Francesco Corsini, MD, ${ }^{2}$ Umberto Rozzanigo, MD, ${ }^{3}$ \\ Franco Chioffi, MD, ${ }^{2}$ and Silvio Sarubbo, MD, $\mathrm{PhD}^{2}$
}

${ }^{1}$ Center for Mind/Brain Sciences, University of Trento; and 2Division of Neurosurgery, Structural and Functional Connectivity Lab Project, and ${ }^{3}$ Department of Radiology, Neuroradiology Unit, "S. Chiara" Hospital, Trento, Italy

\begin{abstract}
OBJECTIVE Resting-state functional MRI (rs-fMRI) represents a promising and cost-effective alternative to task-based $\mathrm{fMRI}$ for presurgical mapping. However, the lack of clinically streamlined and reliable rs-fMRI analysis tools has prevented wide adoption of this technique. In this work, the authors introduce an rs-fMRI processing pipeline (ReStNeuMap) for automatic single-patient rs-fMRI network analysis.
\end{abstract}

METHODS The authors provide a description of the rs-fMRI network analysis steps implemented in ReStNeuMap and report their initial experience with this tool after performing presurgical mapping in 6 patients. They verified the spatial agreement between rs-fMRI networks derived by ReStNeuMap and localization of activation with intraoperative direct electrical stimulation (DES).

RESULTS The authors automatically extracted rs-fMRI networks including eloquent cortex in spatial proximity with the resected lesion in all patients. The distance between DES points and corresponding rs-fMRI networks was less than $1 \mathrm{~cm}$ in $78 \%$ of cases for motor, $100 \%$ of cases for visual, $87.5 \%$ of cases for language, and $100 \%$ of cases for speech articulation mapping.

CONCLUSIONS The authors' initial experience with ReStNeuMap showed good spatial agreement between presurgical rs-fMRI predictions and DES findings during awake surgery. The availability of the rs-fMRI analysis tools for clinicians aiming to perform noninvasive mapping of brain functional networks may extend its application beyond surgical practice. https://thejns.org/doi/abs/10.3171/2018.4.JNS18474

KEYWORDS brain surgery; brain mapping; presurgical planning; resting state; functional magnetic resonance imaging; diagnostic technique

$\mathrm{R}$ ESTING-STATE functional MRI (rs-fMRI) is currently an established technique for extraction of brain networks. ${ }^{4,17}$ Multiple studies have demonstrated good overlap between the areas of fMRI activation elicited by motor, language, and visual tasks and the topography of the corresponding rs-fMRI networks. ${ }^{7,30,34}$ In comparison with task-based fMRI, rs-fMRI offers multiple advantages, including 1) reduced image acquisition time due to the possibility of detecting multiple networks from 1 data set; 2) no need for specific hardware and software for delivery of the task-related stimuli; 3) no need for dedicated personnel for evaluating patients' cognitive status, select- ing the tasks, and assessing task performance; and 4) the possibility of studying noncooperative populations, such as neurologically impaired patients and children, who may not be adequately compliant for task-based fMRI, as well as nonresponsive patients (e.g., coma or vegetative state).

These advantages make rs-fMRI a promising and costeffective alternative to task-based fMRI for presurgical mapping of patients undergoing surgery for brain tumor or vascular malformation in the spatial proximity of eloquent cortical and subcortical areas. ${ }^{20}$ Moreover, this noninvasive neuroimaging technique could be particularly useful in monitoring the functional reorganization of patients

ABBREVIATIONS DES = direct electrical stimulation; $\mathrm{GOF}=$ goodness of fit; ICA = independent component analysis; $L G G=$ low-grade glioma; $Q \mathrm{QC}=$ quality control; ReStNeuMap = resting-state neurosurgical mapping; rs-fMRI = resting-state functional MRI.

ACCOMPANYING EDITORIALS AND RESPONSE See pp 757-763. DOIs: 10.3171/2018.5.JNS181057; 10.3171/2018.5.JNS181058; and 10.3171/2018.6.JNS181568. SUBMITTED February 20, 2018. ACCEPTED April 17, 2018.

INCLUDE WHEN CITING Published online October 26, 2018; DOI: 10.3171/2018.4.JNS18474. 
with longer survival (e.g., those with low-grade gliomas [LGGs]) in order to evaluate possible reinterventions. ${ }^{38}$

However, the wide adoption of rs-fMRI in surgical and postsurgical practice is limited by the lack of clinically streamlined tools for quality control (QC), preprocessing, and rs-fMRI network analysis. Using rs-fMRI software freely available to the neuroimaging community (e.g., AFNI, SPM, and FSL), it is possible to perform preoperative localization of critical cortical eloquent area for presurgical planning. ${ }^{18,31,40}$ However, the setup of a clinical rsfMRI analysis pipeline requires considering the following methodological and practical issues. 1) Multiple data QC parameters should be provided, including a) the determination of rs-fMRI volume outliers due to head motion, physiology, or hardware; ${ }^{16}$ b) the quality of the coregistration between functional and structural MR images; ${ }^{26}$ and c) the accuracy of the T1-weighted image segmentation, to help with a correct interpretation of the results. ${ }^{25}$ 2) For rs-fMRI network extraction, it is preferable to use an unsupervised technique, such as independent component analysis (ICA), to take into account the anatomical variability in localization of functional areas across patients. ${ }^{19}$ 3) The entire data analysis process should be completely automated, including the identification of the rs-fMRI networks of interest, to streamline the clinical workflow, which allows the pipeline to be run by an imaging technologist with no specific fMRI expertise and then evaluated by a clinician. Furthermore, it is advisable to investigate the reliability of the rs-fMRI analysis pipeline by comparing the spatial correspondence between the preoperative rs-fMRI networks and intraoperative localization during direct electrical stimulation (DES) of corresponding functions in awake surgery.

To the best of our knowledge, only one analysis pipeline specifically designed for presurgical mapping with rs-fMRI has been proposed. ${ }^{14}$ Many processing steps are automated, and an option for automatic labeling of rsfMRI networks is available with this tool. However, the model that the authors used does not automatically extract all the raw DICOM image parameters that are needed for preprocessing, and the only QC parameter is head motion estimation. Furthermore, its results have been validated only for language mapping. ${ }^{21}$ In this study, we introduce a pipeline (resting-state neurosurgical mapping, ReStNeuMap) for rs-fMRI data analysis designed to meet all of the aforementioned surgical and nonsurgical needs. We also assessed the agreement between its results and those of DES for motor, language, visual, and speech articulation mapping in a series of patients.

\section{Methods}

\section{Pipeline Implementation}

ReStNeuMap runs within MATLAB (version 8.5.0, MathWorks) and is currently available for Mac OS X (version 10.11.5) and Linux (Ubuntu 16.4) operating systems. The data analysis is based on SPM12 (fil.ion.ucl.ac.uk/spm/ software/spm12/) original packages and plugins for preprocessing and MELODIC-FSL (version 3.14; fsl.fmrib.ox.ac. $\mathrm{uk} / \mathrm{fsl} /$ fslwiki/MELODIC) for rs-fMRI network extraction. ${ }^{5}$ The chart in Fig. 1 shows the workflow of the pipeline with the indication of the software package used in each step.

\section{Preprocessing}

The user is asked to select only the folders in which the raw anatomical T1-weighted and rs-fMRI DICOM images are stored. From this point onward, the pipeline is automatized. A series of standard preprocessing steps is automatically performed, as listed in Supplementary Table 1.8,24,35

\section{Quality Control}

In clinical rs-fMRI studies, it is critical to assess (quantitatively when possible) and remove head motion, signal spikes, and image artifacts to guarantee the reliability and proper interpretation of the rs-fMRI mapping results. For this reason we included in our pipeline the ArtRepair toolbox (version 5b; cibsr.stanford.edu/tools/human-brainproject/artrepair-software.html), which can automatically detect and remove noisy volumes in rs-fMRI data series (Supplementary Fig. 1). ${ }^{23}$ However, if more than $20 \%$ of the volumes are classified as outliers, ArtRepair may not succeed in removing head motion and spike artifacts. ${ }^{23}$

Since rs-fMRI networks are overlaid onto the patient's anatomical images for better localization of functional activation, it is also important to verify the quality of the coregistration between the rs-fMRI and T1-weighted MR images. To this purpose, at the end of the preprocessing, our pipeline visualizes the coregistered rs-fMRI and T1weighted images (Supplementary Fig. 1b). We also recommend visually checking the segmentation quality of the T1-weighted images because the incorrect regression of the rs-fMRI signal of interest can result in inaccurate localization. ${ }^{25}$ Furthermore, for the segmentation of the T1weighted images, transformations to align these images to common Montreal Neurological Institute space are calculated. Since this information is used, as explained in the next section, to automatically label the rs-fMRI networks, good-quality segmentation is necessary to obtain reliable results with ReStNeuMap. In our experience, the coregistration and segmentation are accurate with small lesions. In cases of brain tumors that greatly distort brain anatomy, it could be necessary to run the pipeline after segmenting out the lesion and finally overlaying the rs-fMRI networks on the original T1-weighted images.

\section{Resting-State fMRI Network Calculations}

In ReStNeuMap, we use ICA for rs-fMRI network extraction. This technique decomposes the rs-fMRI signal in $n$ time series statistically independent of each other (components). Then, each voxel time series is expressed as the linear combination of these $n$ components. The coefficient of each component is assigned statistical significance, and the set of voxels with statistical significance below a defined threshold (e.g., p < 0.001) compose the componentassociated network. To automatically identify the rs-fMRI networks, we provide a matching template procedure. Specifically, we measure the amount of spatial overlap between each network determined by ICA with the network provided by an atlas representing the functional system that needs to be mapped. ${ }^{33,39}$ We quantify this overlap in patient space by measuring the goodness of fit (GOF). ${ }^{37}$ The optimal number of components in which the rs-fMRI signal is to be split to identify the networks can vary across 


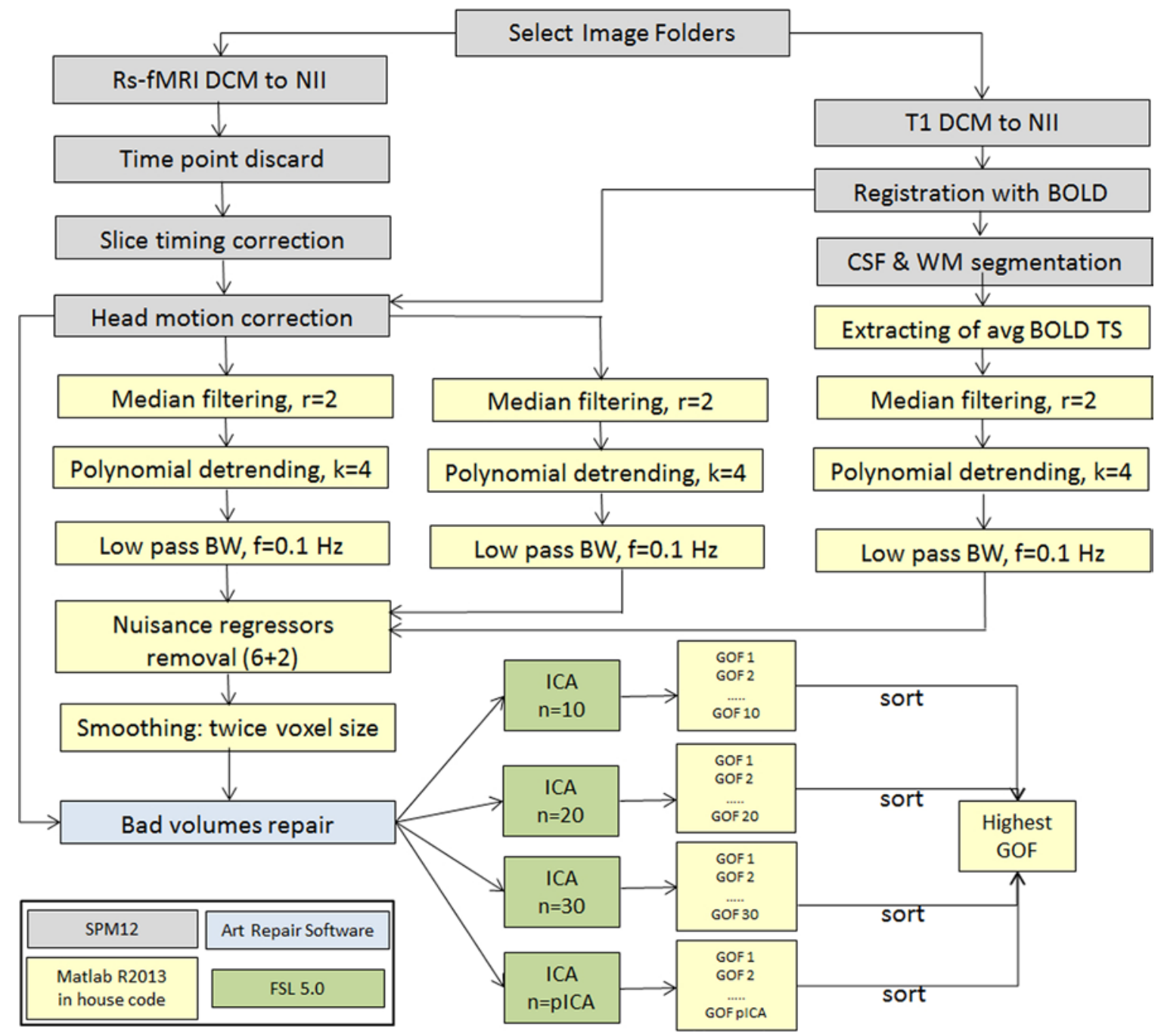

FIG. 1. Flowchart of the rs-fMRI data analysis pipeline implemented in ReStNeuMap. Avg = average; $B W=$ bandwidth; $C S F=$ cerebrospinal fluid; DCM = DICOM format; NII = nifti format; $\mathrm{WM}=$ white matter. Figure is available in color online only.

patients. ${ }^{1}$ Therefore, we run ICA 4 times with the number of components set to $10,20,30$, and a value determined by probabilistic ICA implemented in MELODIC-FSL. For each network that needs to be identified, we select the one with the highest GOF with the corresponding atlas network across all ICA settings. The tool provides 3 predetermined values $(\mathrm{z}=2.5,3.0$, and 3.5$)$ with voxel cluster size $>10$ to threshold the rs-fMRI networks and also gives the user the possibility to apply any threshold.

\section{Case Series Demonstration}

We performed presurgical mapping using ReStNeuMap in 6 patients who underwent asleep-awake-asleep resection of LGGs $(n=4)$ and cavernous angiomas $(n=2)$ with DES (7-mm bipolar probe; $60 \mathrm{~Hz}, 1 \mathrm{msec}$; 2- to 4-mA intensity range) to extract the rs-fMRI networks of interest for preoperative planning. Written informed consent was obtained from all patients included in this series. Table 1 lists the clinical information for each case. Each patient underwent presurgical MRI on a clinical 1.5-T scanner
(GE Healthcare); 3D T1-weighted anatomical images and 2D blood oxygen level-dependent echo planar functional images were acquired with the patient lying in the scanner at rest, keeping his or her eyes open. In Supplementary

TABLE 1. Clinical and demographic data of the patients in whom presurgical mapping was performed

\begin{tabular}{ccll}
\hline Case No. & Age (yrs), Sex & Tumor Location & \multicolumn{1}{c}{ Histology } \\
\hline 1 & $18, \mathrm{M}$ & Rt occipital & Grade I glioma \\
\hline 2 & $45, \mathrm{M}$ & $\begin{array}{c}\text { Rt supplementary } \\
\text { motor area }\end{array}$ & $\begin{array}{c}\text { Grade II oligodendro- } \\
\text { glioma }\end{array}$ \\
\hline 3 & $37, \mathrm{M}$ & Rt frontal & $\begin{array}{c}\text { Grade III anaplastic } \\
\text { astrocytoma }\end{array}$ \\
\hline 4 & $39, \mathrm{~F}$ & Lt frontal & Cavernoma \\
\hline 5 & $37, \mathrm{~F}$ & Lt frontal & Cavernoma \\
\hline 6 & $36, \mathrm{M}$ & Lt temporal & Grade II astrocytoma \\
\hline
\end{tabular}

All patients were right-handed. 
Case 1

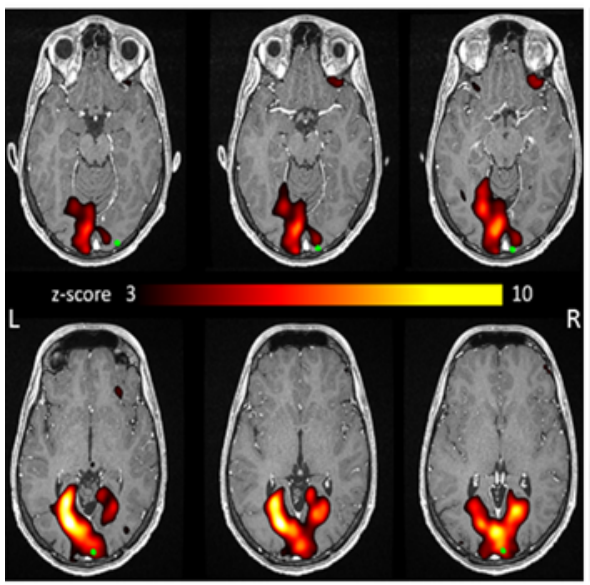

Case 4

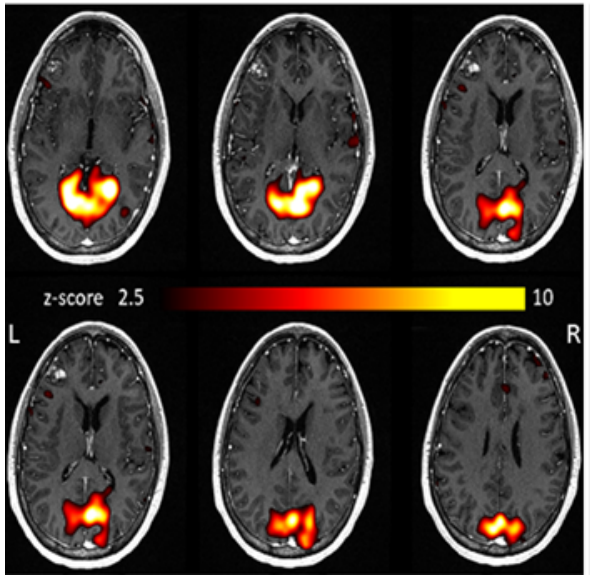

Case 2

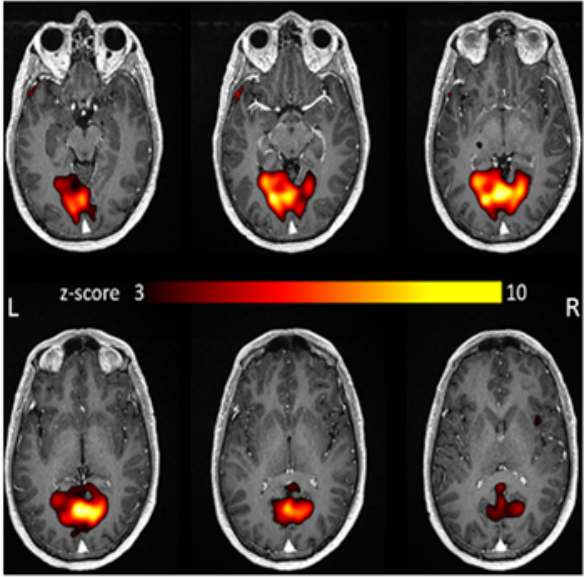

Case 5

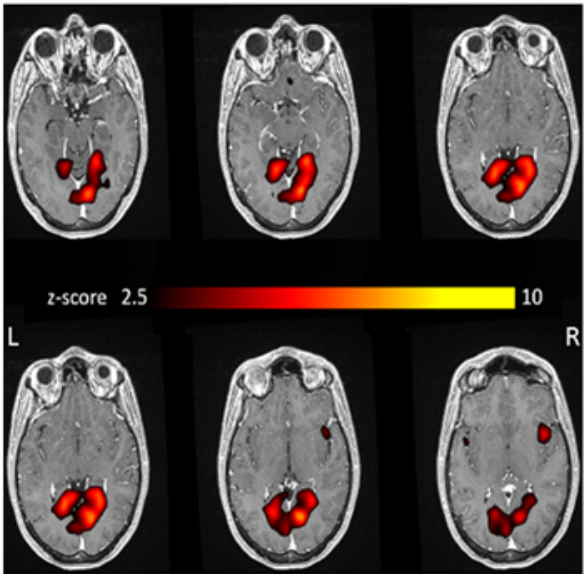

Case 3

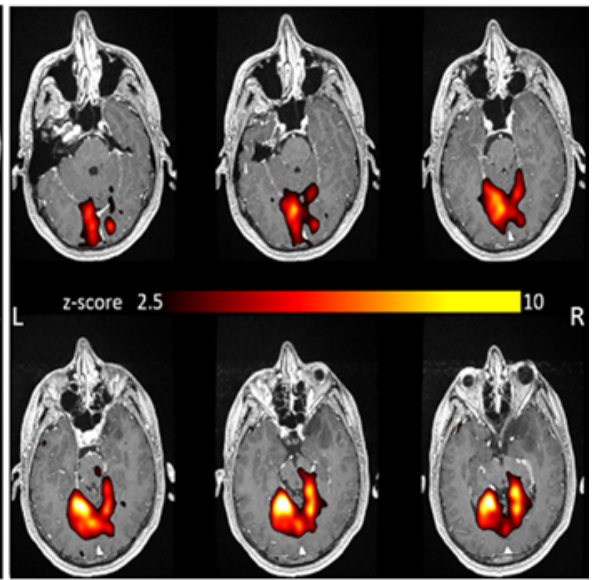

Case 6

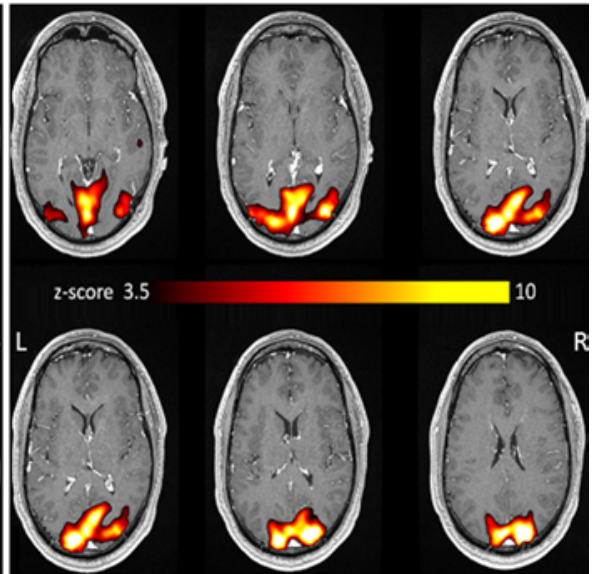

FIG. 2. Visual mapping. Axial rs-fMRI visual networks derived using ReStNeuMap for each of the 6 cases analyzed for this study. The statistical threshold (z-score) applied on the automatically chosen component representing the visual network is reported. For case 1, in which intraoperative mapping of the inferior left visual quadrant was performed, the intraoperative visual activation sites (green circles) detected with DES are shown. Figure is available in color online only.

Table 2 we report the main image acquisition parameters for these 2 sequences.

\section{Comparison of rs-fMRI and DES}

A total of 27 positive ( 8 motor, 8 language, 5 visual, and 6 speech articulation) cortical sites were collected during cortical DES. The cortical sites demonstrated by DES were recorded after surgery on the preoperative T1weighted 3D MR images by the same neurosurgeon (S.S.). We did not mark the activation sites within the navigation platform because of the well-known issues regarding errors due to brain shift during tumor resection that can significantly impact the accuracy of neuronavigation. . $^{27,29}$ Instead, after the resection, we matched the topographical information of the picture with the axial, sagittal, and coronal brain extracted T1-weighted 3D reconstructions, as previously reported. ${ }^{32,36}$ For each patient, the shortest distance of each stimulation point from the rs-fMRI network representing the corresponding functional system (e.g., anomia vs language network) was measured. We calcu- lated the sensitivity of motor, language, visual, and speech articulation rs-fMRI networks as the percentage of corresponding DES points with distance less than $1 \mathrm{~cm} .{ }^{6,28,30}$

\section{Results}

The pipeline execution time on a Macintosh computer (version 10.11.5, processor Intel icore $7.4 \mathrm{GHz}, 16 \mathrm{~GB}$ RAM at $1687 \mathrm{MHz}$ ) to process one patient's data set $(0.25-$ $\mathrm{mm}^{3}$ voxels, 5 minutes for acquisition of structural images, $64 \mathrm{~mm}^{3} \times 275$ brain scans, 10 minutes for acquisition of rs-fMR images) was between 25 and 30 minutes. For all patients, the rs-fMRI data were not affected by excessive outliers (i.e., $<20 \%$ of the volumes), and the quality of the segmentation and coregistration was deemed adequate. Supplementary Table 3 lists the following for each case and each network: 1) the number of components into which the signal was decomposed to provide the highest GOF; 2) the selected component with its corresponding GOF value; and 3) the threshold applied to each rs-fMRI network. Below, we report the results obtained for visual, 
Case 1

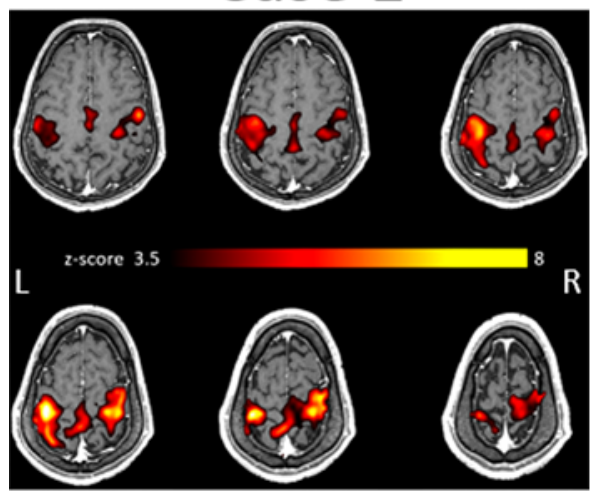

Case 4

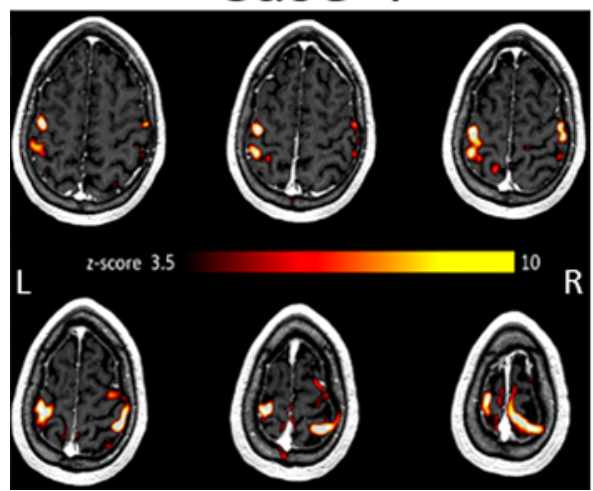

Case 2

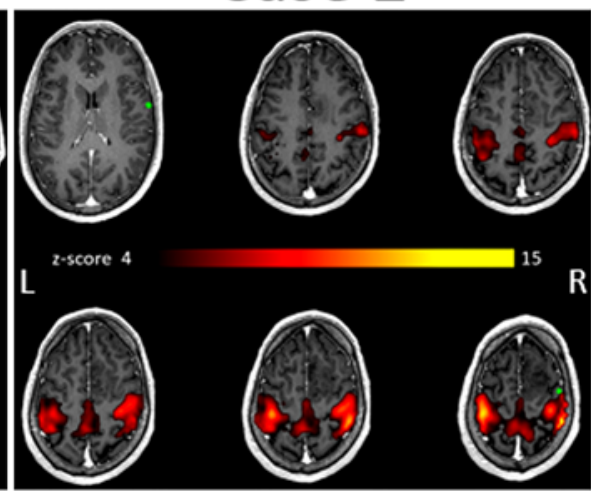

Case 5

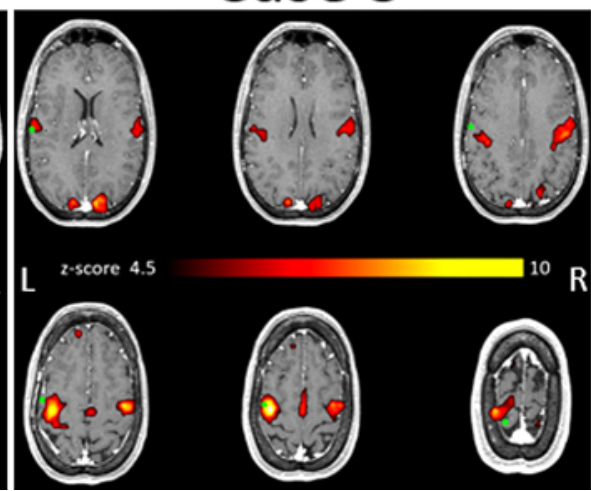

Case 3

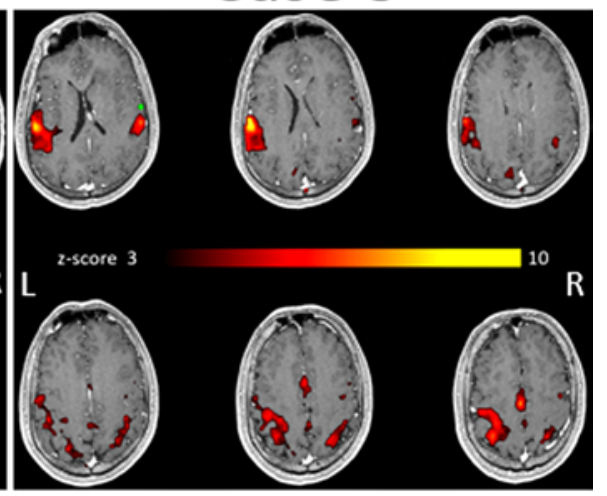

Case 6

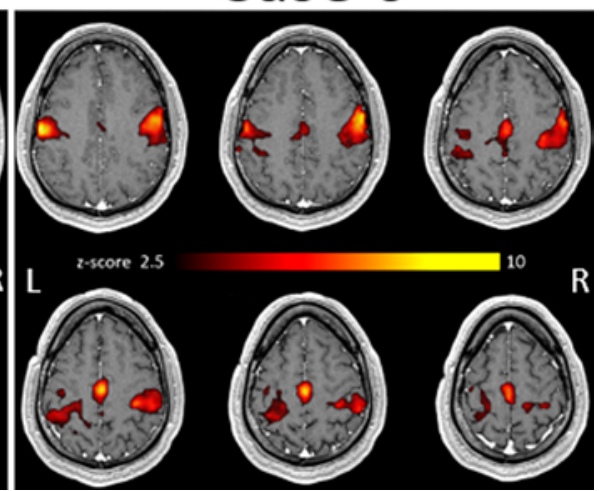

FIG. 3. Motor mapping. Axial rs-fMRI motor networks derived using ReStNeuMap for each of the 6 cases analyzed for this study. The statistical threshold (z-score) applied on the automatically chosen component representing the motor network is reported. For cases 2, 3, and 5, in which intraoperative motor mapping was performed, the intraoperative motor activation sites (green circles) detected with DES are shown. Figure is available in color online only.

motor, and language presurgical rs-fMRI networks and, when available, the corresponding intraoperative DES positive sites.

\section{Visual Mapping}

For all 6 cases, ReStNeuMap identified a network including expected areas of the visual cortex (Fig. 2). The patient in case 1 underwent intraoperative mapping of the inferior left visual quadrant because of the spatial proximity of the lesion to this area, and $100 \%$ of the DES positive sites were identified with a distance within $1 \mathrm{~cm}$ from the rs-fMRI maps.

\section{Motor Mapping}

Intraoperative motor mapping was performed for lesions located in the supplementary motor or frontal areas (cases 2, 3, and 5; Fig. 3). In 78\% of the recorded positive sites, there was spatial overlap $(<1 \mathrm{~cm})$ with the preoperative motor rs-fMRI network. In all 6 cases, ReStNeuMap provided motor maps with expected topography.

\section{Language Mapping}

Intraoperative language mapping was performed in cases 4,5 , and 6 , who were right-handed and had a lesion located in the frontal or temporal lobe of the left hemi- sphere (Fig. 4). All but one (87.5\%) DES recorded site for language activation were located less than $1 \mathrm{~cm}$ from the corresponding rs-fMRI language network. In all 6 (righthanded) patients, rs-fMRI language networks were mostly localized in the left hemisphere and included areas known to be involved in language (superior temporal, inferior frontal, dorsolateral prefrontal, and supplementary premotor cortex).

\section{Speech Articulation Mapping}

Speech arrest was recorded in the ipsilateral hemisphere (3 right and 3 left) as the first DES point in all 6 cases, and there was $100 \%$ overlap between these sites and preoperative determined speech articulation networks (see Supplementary Fig. 2).

\section{Discussion}

We have described a pipeline for processing single-patient rs-fMRI data for surgical and clinical mapping, and we reported our initial experience with 6 patients referred for resection of 2 different brain lesions.

With respect to similar pipelines that have been proposed, ${ }^{14}$ our system represents a significant advancement for the following reasons. 1) The analysis settings depend- 


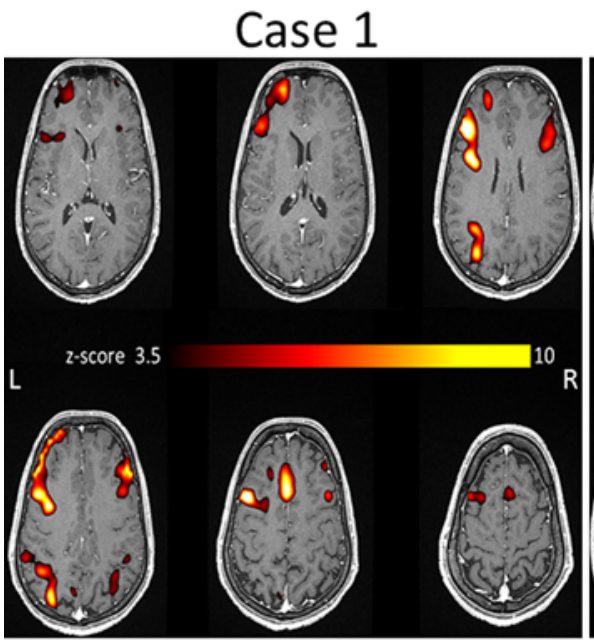

Case 4

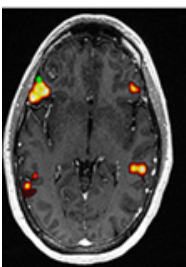

2-score 4
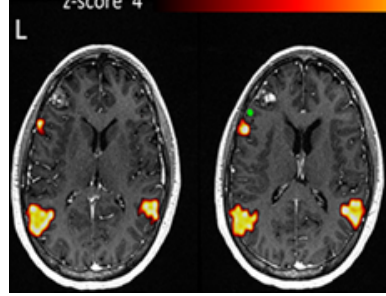

10
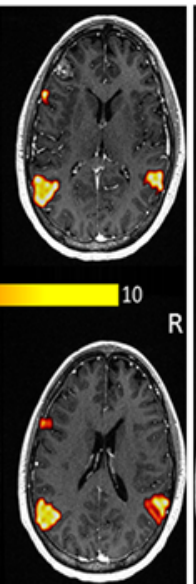

Case 2

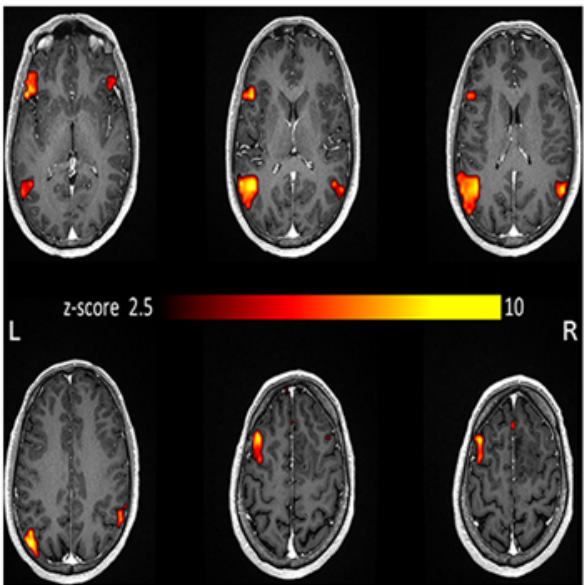

Case 5

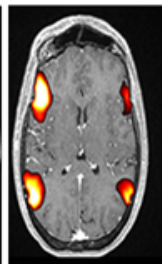

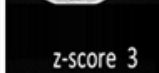

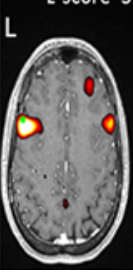

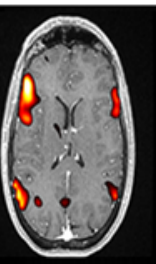

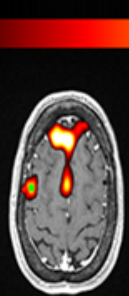

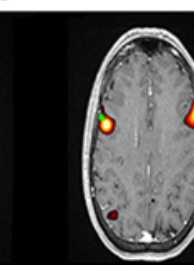
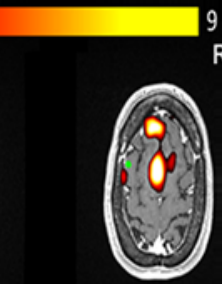

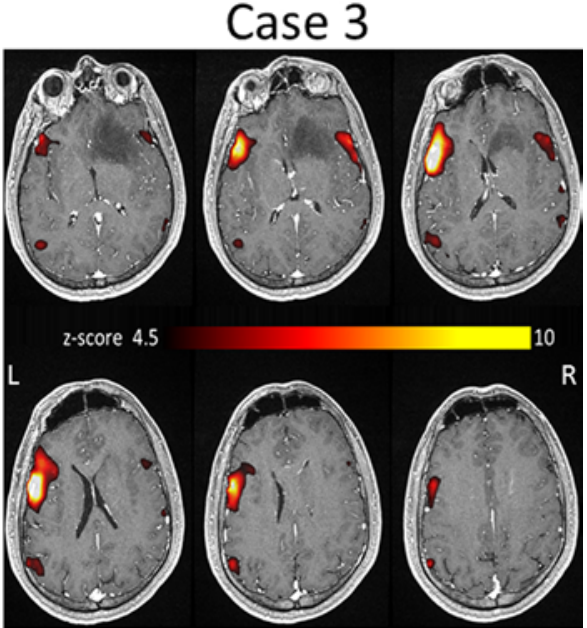

Case 6

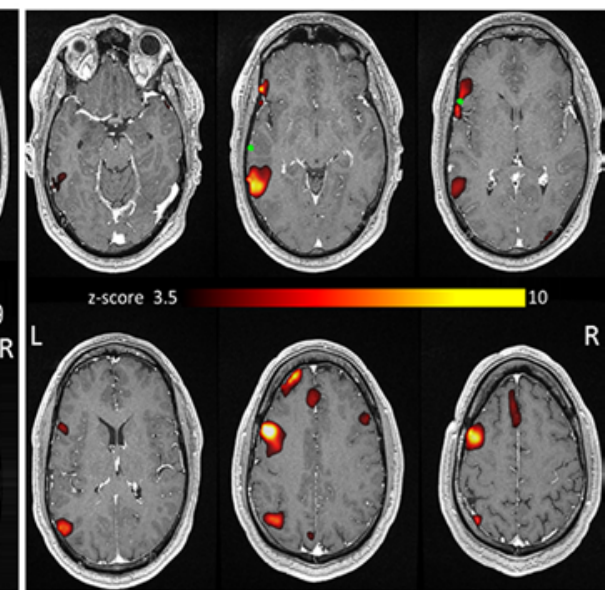

FIG. 4. Language mapping. Axial rs-fMRI language networks derived using ReStNeuMap for each of the 6 cases analyzed for this study. The statistical threshold (z-score) applied on the automatically chosen component representing the motor network is reported. For cases 4,5 , and 6 , in which intraoperative language mapping was performed, the intraoperative language activation sites (green circles) detected with DES are shown. Figure is available in color online only.

ing on DICOM image parameters are automatically read from the raw data, minimizing manual user intervention. 2) The pipeline includes the evaluation of QC parameters beyond head motion estimation, such as spikes in the raw blood oxygen level-dependent signal, image coregistration, and segmentation. 3) The application of the pipeline in 6 patients demonstrated a good degree of spatial concordance between the localization of multiple rs-fMRI networks (motor, language, visual, and speech articulation) and the cortical sites that, when stimulated by intraoperative DES, impaired the corresponding function., ${ }^{9,21,30}$

Signal spikes can affect the detection of the rs-fMRI connectivity pattern in the brain. Head motion is one, but not the only, cause of signal spikes. ${ }^{16}$ Therefore, it is critical to check for these spikes separately from head motion. Indeed, by integrating the ArtRepair in the pipeline, we are able to detect and repair volume outliers due to head motion and spikes. With regard to the comparison between rs-fMRI and DES results, to the best of our knowledge, this is the first study that validates the results of rs-fMRI for mapping speech articulation. This result is relevant be- cause, for intraoperative mapping with DES, speech arrest, the deficit used to map speech articulation, is routinely adopted as the first negative control task to define the current amplitude threshold for the entire cortical-subcortical mapping. ${ }^{13,22}$

More generally, the noninvasive neuroimaging brain mapping tool described in this work has the potential to 1) be used not as an alternative but as a complementary tool to DES in awake surgery to reduce the time and number of cortical DES attempts for intraoperative localization of critical functions (in particular, speech arrest or motor); and 2) track the functional reorganization of rs-fMRI networks during follow-up at the whole-brain level, such as in LGG patients. ${ }^{10}$

We acknowledge that despite the advantages of the described toolbox, some limitations exist in its current implementation. First, the spatial template-matching procedure allows identifying the networks most commonly needed for presurgical mapping. However, it could be of clinical interest also to assess the connectivity of other networks, such as the default mode, which has been shown to 
be affected by brain tumor regardless of its localization. ${ }^{12}$ Second, in patients with focal brain lesions, the altered coupling between neuronal activity and blood hemodynamics (neurovascular uncoupling) can result in false-negative findings on rs-fMRI activation maps. ${ }^{3} \mathrm{~A}$ QC metric to assess neurovascular uncoupling is not yet available, although the regional homogeneity of the rs-fMRI signal could be used for this purpose. ${ }^{2}$ Third, we did not include in the preprocessing a step for correction of geometric distortion on the echo planar images, which could affect the localization of functional activation, especially at ultrahigh magnetic fields ( $\geq 3 \mathrm{~T}$ ). We plan to add this feature in future versions of the pipeline. ${ }^{15}$ Finally, we did not check the consistency of the rs-fMRI networks identified in our pipeline with the activation of the same network obtained with task-based fMRI data or with different established methods for rs-fMRI data analysis (e.g., seed based). However, we found good concordance between rs-fMRI network localization and the results of DES, ${ }^{11}$ the gold standard for safe resections in critical areas. Nevertheless, due to the small number of cases in which the pipeline was tested, ReStNeuMap requires further validation in a large, multisite study.

\section{Conclusions}

We present a software tool aimed at facilitating the derivation of rs-fMRI brain networks for surgical and clinical purposes. We expect that the availability of an automated and reliable pipeline for rs-fMRI data analysis will expand its use to specialties beyond neurosurgical practice (e.g., neurology, physical medicine, and radiation therapy) and outside academic centers. To this aim we make ReStNeuMap freely available for download (https:/github.com/ CIMeC-MRI-Lab/ReStNeuMap).

\section{Acknowledgments}

This work was supported by the Caritro Foundation within the "Bando 2015 per progetti di ricerca scientifica svolti da giovani ricercatori" (University of Trento, Italy).

\section{References}

1. Abou-Elseoud A, Starck T, Remes J, Nikkinen J, Tervonen O, Kiviniemi V: The effect of model order selection in group PICA. Hum Brain Mapp 31:1207-1216, 2010

2. Agarwal S, Sair HI, Pillai JJ: The resting-state functional magnetic resonance imaging regional homogeneity metricsKendall's coefficient of concordance-regional homogeneity and coherence-regional homogeneity-are valid indicators of tumor-related neurovascular uncoupling. Brain Connect 7:228-235, 2017

3. Agarwal S, Sair HI, Yahyavi-Firouz-Abadi N, Airan R, Pillai JJ: Neurovascular uncoupling in resting state fMRI demonstrated in patients with primary brain gliomas. J Magn Reson Imaging 43:620-626, 2016

4. Azeez AK, Biswal BB: A review of resting-state analysis methods. Neuroimaging Clin N Am 27:581-592, 2017

5. Beckmann CF, Smith SM: Probabilistic independent component analysis for functional magnetic resonance imaging. IEEE Trans Med Imaging 23:137-152, 2004

6. Bizzi A, Blasi V, Falini A, Ferroli P, Cadioli M, Danesi U, et al: Presurgical functional MR imaging of language and motor functions: validation with intraoperative electrocortical mapping. Radiology 248:579-589, 2008
7. Branco P, Seixas D, Deprez S, Kovacs S, Peeters R, Castro SL, et al: Resting-state functional magnetic resonance imaging for language preoperative planning. Front Hum Neurosci 10:11, 2016

8. Chen Z, Calhoun V: Effect of spatial smoothing on task fMRI ICA and functional connectivity. Front Neurosci 12:15, 2018

9. Cochereau J, Deverdun J, Herbet G, Charroud C, Boyer A, Moritz-Gasser S, et al: Comparison between resting state fMRI networks and responsive cortical stimulations in glioma patients. Hum Brain Mapp 37:3721-3732, 2016

10. Corrivetti F, Herbet G, Moritz-Gasser S, Duffau H: Prosopagnosia induced by a left anterior temporal lobectomy following a right temporo-occipital resection in a multicentric diffuse low-grade glioma. World Neurosurg 97:756.e1-756. e5, 2017

11. Duffau H: Surgery of low-grade gliomas: towards a 'functional neurooncology'. Curr Opin Oncol 21:543-549, 2009

12. Esposito R, Mattei PA, Briganti C, Romani GL, Tartaro A, Caulo M: Modifications of default-mode network connectivity in patients with cerebral glioma. PLoS One 7:e40231, 2012

13. Freyschlag CF, Duffau H: Awake brain mapping of cortex and subcortical pathways in brain tumor surgery. J Neurosurg Sci 58:199-213, 2014

14. Huang H, Ding Z, Mao D, Yuan J, Zhu F, Chen S, et al: PreSurgMapp: a MATLAB toolbox for presurgical mapping of eloquent functional areas based on task-related and restingstate functional MRI. Neuroinformatics 14:421-438, 2016

15. Jezzard P: Correction of geometric distortion in fMRI data. Neuroimage 62:648-651, 2012

16. Jo HJ, Gotts SJ, Reynolds RC, Bandettini PA, Martin A, Cox RW, et al: Effective preprocessing procedures virtually eliminate distance-dependent motion artifacts in resting state FMRI. J Appl Math 2013:935154, 2013

17. Khalili-Mahani N, Rombouts SA, van Osch MJ, Duff EP, Carbonell F, Nickerson LD, et al: Biomarkers, designs, and interpretations of resting-state fMRI in translational pharmacological research: a review of state-of-the-art, challenges, and opportunities for studying brain chemistry. Hum Brain Mapp 38:2276-2325, 2017

18. Kokkonen SM, Nikkinen J, Remes J, Kantola J, Starck T, Haapea M, et al: Preoperative localization of the sensorimotor area using independent component analysis of restingstate fMRI. Magn Reson Imaging 27:733-740, 2009

19. Kong NW, Gibb WR, Tate MC: Neuroplasticity: insights from patients harboring gliomas. Neural Plast 2016: 2365063,2016

20. Lee MH, Miller-Thomas MM, Benzinger TL, Marcus DS, Hacker CD, Leuthardt EC, et al: Clinical resting-state fMRI in the preoperative setting: are we ready for prime time? Top Magn Reson Imaging 25:11-18, 2016

21. Lu J, Zhang H, Hameed NUF, Zhang J, Yuan S, Qiu T, et al: An automated method for identifying an independent component analysis-based language-related resting-state network in brain tumor subjects for surgical planning. Sci Rep 7:13769, 2017

22. Mandonnet E, Winkler PA, Duffau H: Direct electrical stimulation as an input gate into brain functional networks: principles, advantages and limitations. Acta Neurochir (Wien) 152:185-193, 2010

23. Mazaika PK, Hoeft F, Glover GH, Reiss AL: Methods and software for fMRI analysis of clinical subjects. Neuroimage 47:S58, 2009

24. Minati L, Chan D, Mastropasqua C, Serra L, Spanò B, Marra $\mathrm{C}$, et al: Widespread alterations in functional brain network architecture in amnestic mild cognitive impairment. J Alzheimers Dis 40:213-220, 2014

25. Murphy K, Birn RM, Handwerker DA, Jones TB, Bandettini 
PA: The impact of global signal regression on resting state correlations: are anti-correlated networks introduced? Neuroimage 44:893-905, 2009

26. Nennig E, Heiland S, Rasche D, Sartor K, Stippich C: Functional magnetic resonance imaging for cranial neuronavigation: methods for automated and standardized data processing and management. A technical note. Neuroradiol J 20:159-168, 2007

27. Nimsky C, Ganslandt O, Hastreiter P, Wang R, Benner T, Sorensen AG, et al: Intraoperative diffusion-tensor MR imaging: shifting of white matter tracts during neurosurgical procedures-initial experience. Radiology 234:218-225, 2005

28. Roessler K, Donat M, Lanzenberger R, Novak K, Geissler A, Gartus A, et al: Evaluation of preoperative high magnetic field motor functional MRI (3 Tesla) in glioma patients by navigated electrocortical stimulation and postoperative outcome. J Neurol Neurosurg Psychiatry 76:1152-1157, 2005

29. Romano A, D'Andrea G, Calabria LF, Coppola V, Espagnet CR, Pierallini A, et al: Pre- and intraoperative tractographic evaluation of corticospinal tract shift. Neurosurgery 69:696-705, 2011

30. Rosazza C, Aquino D, D'Incerti L, Cordella R, Andronache $\mathrm{A}$, Zacà D, et al: Preoperative mapping of the sensorimotor cortex: comparative assessment of task-based and restingstate FMRI. PLoS One 9:e98860, 2014

31. Sair HI, Yahyavi-Firouz-Abadi N, Calhoun VD, Airan RD, Agarwal S, Intrapiromkul J, et al: Presurgical brain mapping of the language network in patients with brain tumors using resting-state fMRI: comparison with task fMRI. Hum Brain Mapp 37:913-923, 2016

32. Sarubbo S, De Benedictis A, Merler S, Mandonnet E, Balbi S, Granieri E, et al: Towards a functional atlas of human white matter. Hum Brain Mapp 36:3117-3136, 2015

33. Shirer WR, Ryali S, Rykhlevskaia E, Menon V, Greicius MD: Decoding subject-driven cognitive states with whole-brain connectivity patterns. Cereb Cortex 22:158-165, 2012

34. Smith SM, Fox PT, Miller KL, Glahn DC, Fox PM, Mackay $\mathrm{CE}$, et al: Correspondence of the brain's functional architecture during activation and rest. Proc Natl Acad Sci U S A 106: $13040-13045,2009$

35. Soares JM, Magalhães R, Moreira PS, Sousa A, Ganz E, Sampaio A, et al: A hitchhiker's guide to functional magnetic resonance imaging. Front Neurosci 10:515, 2016

36. Tate MC, Herbet G, Moritz-Gasser S, Tate JE, Duffau H: Probabilistic map of critical functional regions of the human cerebral cortex: Broca's area revisited. Brain 137:27732782, 2014

37. Van Dijk KR, Hedden T, Venkataraman A, Evans KC, Lazar
SW, Buckner RL: Intrinsic functional connectivity as a tool for human connectomics: theory, properties, and optimization. J Neurophysiol 103:297-321, 2010

38. Vassal M, Charroud C, Deverdun J, Le Bars E, Molino F, Bonnetblanc F, et al: Recovery of functional connectivity of the sensorimotor network after surgery for diffuse low-grade gliomas involving the supplementary motor area. J Neurosurg 126:1181-1190, 2017

39. Zacà $\mathrm{D}$, Corsini $\mathrm{F}$, Rozzanigo U, Dallabona $\mathrm{M}$, Avesani $\mathrm{P}$, Annichiarico L, et al: Whole-brain network connectivity underlying the human speech articulation as emerged integrating direct electric stimulation, resting state fMRI and tractography. Front Hum Neurosci 12:405, 2018

40. Zhang D, Johnston JM, Fox MD, Leuthardt EC, Grubb RL, Chicoine MR, et al: Preoperative sensorimotor mapping in brain tumor patients using spontaneous fluctuations in neuronal activity imaged with functional magnetic resonance imaging: initial experience. Neurosurgery 65 (6 Suppl):226-236, 2009

\section{Disclosures}

The authors report no conflict of interest concerning the materials or methods used in this study or the findings specified in this paper.

\section{Author Contributions}

Conception and design: Zacà, Jovicich, Corsini, Chioffi, Sarubbo. Acquisition of data: Rozzanigo, Sarubbo. Analysis and interpretation of data: Zacà, Jovicich, Corsini, Chioffi, Sarubbo. Drafting the article: Zacà. Critically revising the article: Zacà, Jovicich, Sarubbo. Reviewed submitted version of manuscript: all authors. Approved the final version of the manuscript on behalf of all authors: Zacà. Administrative/technical/material support: Rozzanigo, Chioffi, Sarubbo. Study supervision: Jovicich, Sarubbo.

\section{Supplemental Information}

Online-Only Content

Supplemental material is available with the online version of the article.

Supplementary Tables and Figures. https://thejns.org/doi/ suppl/10.3171/2018.4.JNS18474.

\section{Correspondence}

Domenico Zacà: domenico.zaca@gmail.com. 